\title{
Payer-Negotiated Prices for Telemedicine Services
}

KEY WORDS: online digital encounter; payer-negotiated price; price transparency; telemedicine; telephone encounter; virtual check-in.

J Gen Intern Med 37(13):3495-7

DOI: $10.1007 / \mathrm{s} 11606-022-07398-4$

(C) The Author(s) under exclusive licence to Society of General Internal Medicine 2022

\section{INTRODUCTION}

Amid the COVID-19 pandemic, insurers broadened eligibility for telemedicine. ${ }^{1}$ Correspondingly, hospitals expanded telemedicine availability, and utilization is expected to persist. ${ }^{2}$ While attention has been paid to statutory Medicare payment rates for telemedicine services, less is known about private payer prices negotiated with healthcare delivery organizations. Understanding private reimbursement may help policymakers determine the potential for telemedicine to enhance care efficiency and equity moving forward. Leveraging recent Centers for Medicare \& Medicaid Services (CMS) requirements for hospitals to disclose payer-negotiated prices for all items and services, ${ }^{3}$ we examined payer-negotiated prices for telemedicine encounters at top-ranked US hospitals.

\section{METHODS}

Using established methods, ${ }^{3}$ we performed a cross-sectional analysis of private payer-negotiated prices of telemedicine services at top US News and World Report (USNWR) hospitals, which are ranked by patient outcomes, care-related indicators, expert opinion, and patient experience. We restricted analysis to each state's top-ranking hospital disclosing telemedicine pricing given their anticipated resources to comply with price disclosure requirements and offer telemedicine services, as well as in-state requirements to deliver telemedicine. We queried each hospital's website to extract payer-negotiated prices for eight telemedicine encounter codes (Figure 1). To compare prices across hospitals, we used established methods ${ }^{3}$ to calculate the ratio between the 90th and 10th percentile median negotiated prices ("acrosscenter ratio") for each service after standardizing for geographic differences in cost of care delivery. To compare prices between payers within hospitals, we calculated the ratio between the 90th and 10th percentile payer-negotiated prices ("within-center

Received October 12, 2021

Accepted January 4, 2022

Published online January 14, 2022 ratio"). Median encounter prices were compared using Wilcoxon rank-sum tests. Data were current as of September 15, 2021. Analyses were performed using R version 4.0.4. Institutional Review Board approval was not required as no patient records were involved.

\section{RESULTS}

Among 48 states with USNWR-ranked hospitals, 32 $(66.7 \%)$ had a ranked hospital that disclosed pricing for at least one telemedicine service with a median USNWR within-state rank of 2.5 (IQR 1.0-6.3). Raw median prices for brief telephone calls (duration: 5-10 min) varied widely (Figure 2). Depending on encounter duration, raw median payer-negotiated prices ranged from $\$ 20(5-10 \mathrm{~min})$ to $\$ 60$ $(21+\mathrm{min})$ for asynchronous online e-visits, and from $\$ 34$ $(5-10 \mathrm{~min})$ to $\$ 85(21+\mathrm{min})$ for synchronous telephone visits (Figure 1). The median payer-negotiated price for brief (5-10 $\mathrm{min}$ ) synchronous telephone visits was significantly higher than the price for synchronous 5-10 $\mathrm{min}$ virtual check-ins (\$34.02 vs. $\$ 21.81, p=0.03)$. Geographic-standardized across-center price ratios ranged from $2.6(21+\mathrm{min})$ to $3.0(5-10 \mathrm{~min})$ for e-visits and 3.2 $(21+\min )$ to $4.5(5-10 \mathrm{~min})$ for telephone visits. Median within-center price ratios ranged from 1.7 (11-20 $\mathrm{min})$ to $1.8(5-10$ and $21+\mathrm{min})$ for e-visits and $2.1(21+\mathrm{min})$ to 2.4 (5-10 $\mathrm{min}$ ) for telephone visits. Subset analyses comparing hospitals on Disproportionate Share Hospital status and rural/urban location revealed no significant differences in telemedicine prices (data not shown).

\section{DISCUSSION}

Two-third of states had at least one USNWR-ranked hospital disclosing payer-negotiated prices for telemedicine services as required by law - namely, negotiated rates for all items and services. ${ }^{3}$ To promote price transparency, CMS could add telemedicine encounters to the 300 shoppable services required to be disclosed in a consumer-friendly display, as is required for in-person clinic visits, rather than cumbersome machine-readable files.

We found wide variation in standardized prices for telemedicine services. For instance, telephone visits varied up to 4.5fold across centers. This was unexpected given the largely commoditized nature of telemedicine input costs and may reflect differences in market power. 

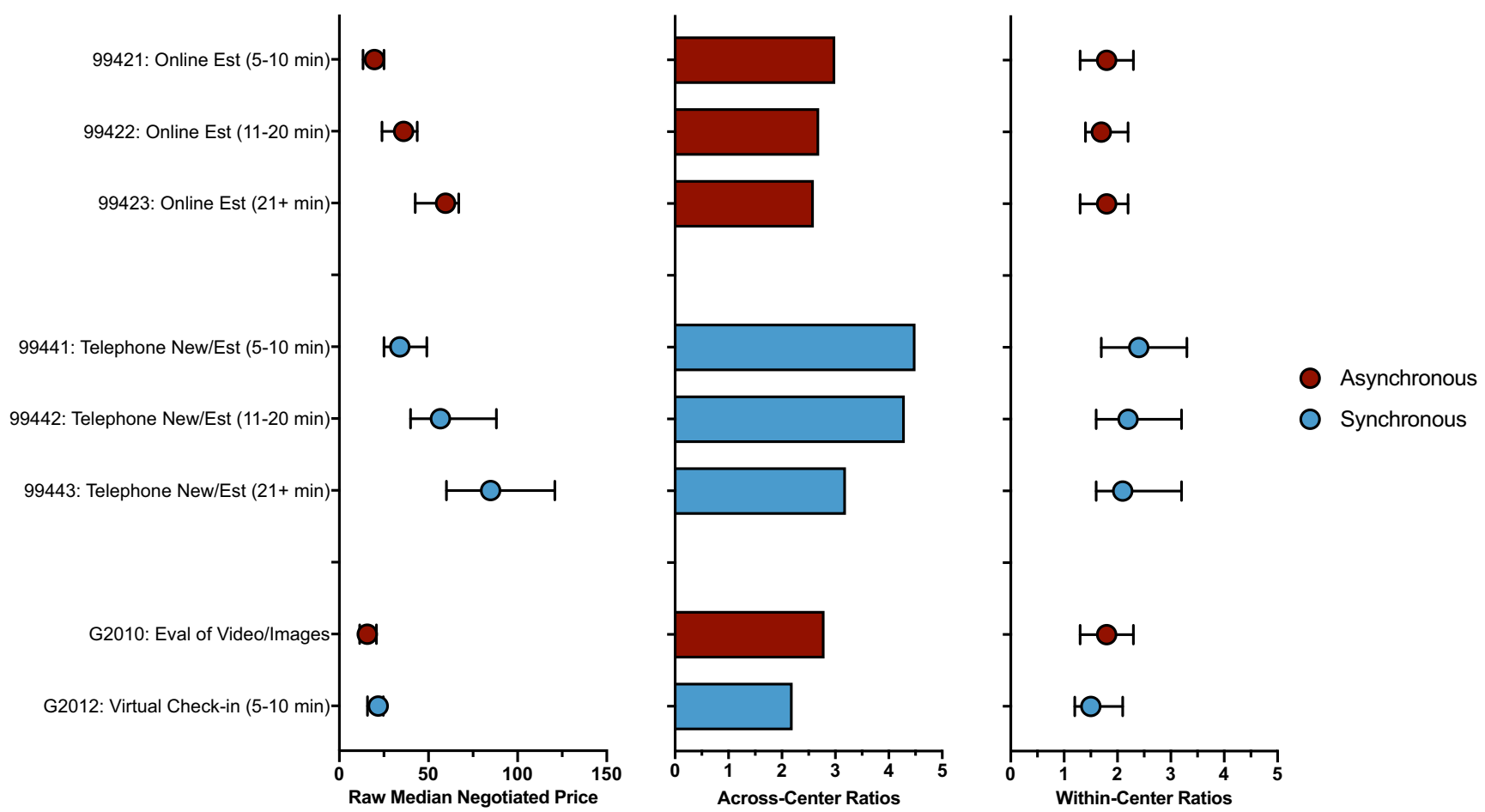

Figure 1 Raw median prices, across-center ratios, and within-center ratios for telemedicine services across the USA. (Left) Raw median prices for all included telemedicine services; bars represent associated interquartile ranges. (Middle)Across-center ratios defined as ratio between the 90th and 10th percentile median payer-specific negotiated prices for each telemedicine service. (Right)Within-center ratios defined as ratio between 90th percentile and 10th percentile prices for each telemedicine service; bars represent associated interquartile ranges.

We found that prices were significantly higher for brief synchronous telephone visits compared to synchronous virtual check-ins. This may unintentionally exacerbate care disparities for socioeconomically vulnerable patients with limited access to broadband Internet or audiovisual communication devices. Congress is considering legislation to expand high-speed Internet access which could mitigate such disparities. ${ }^{4}$ The BidenHarris Administration has also invested in telehealth innovation to support rural and underserved communities. ${ }^{5}$ Continued reimbursement for audio-only telephone visits concomitant with expanded infrastructure for audiovisual visits (i.e., Federally Qualified Health Centers) may lessen disparities. ${ }^{6}$

Our limitations include lack of generalizability given that we selected each state's highest-ranking hospital and may overestimate disclosure rates or prices. Our findings are limited to select telemedicine encounters and may not be generalizable to other telehealth services, such as behavioral health or remote monitoring services.
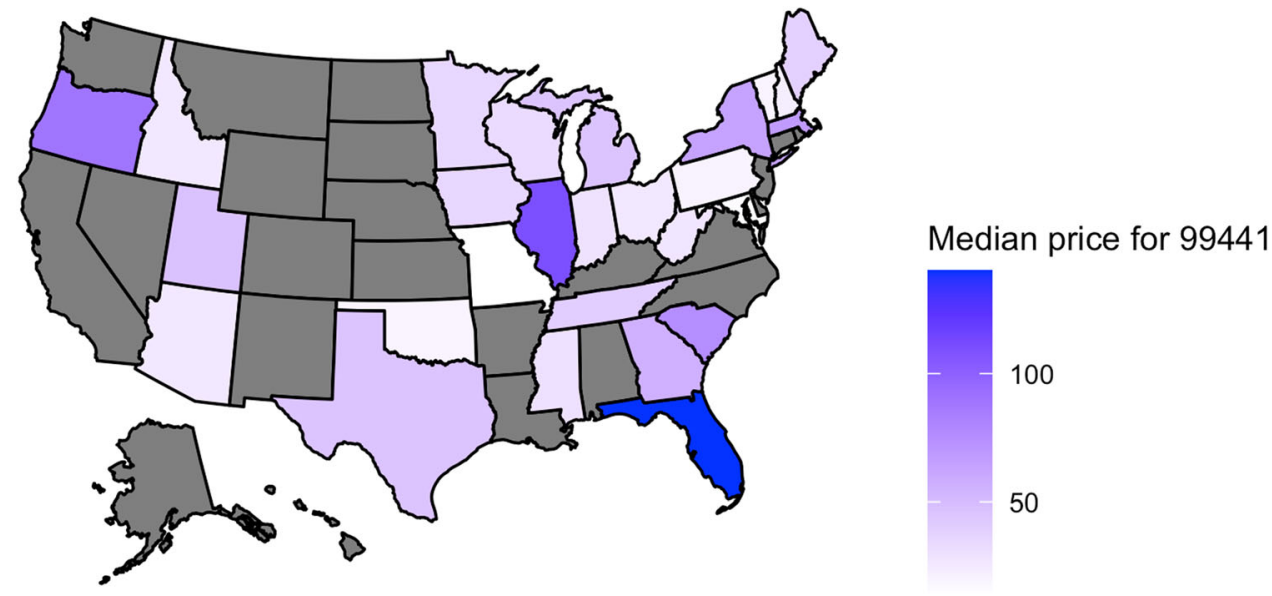

Figure 2. Variation in raw median payer-negotiated prices for brief (5-10 min) telephone calls across the USA. The median payer-negotiated price for the telemedicine CPT code 99441 (Telephone E/M new or established patient, 5-10 min) within the included hospital for each state is shown. Color intensity indicates the magnitude of price. Included hospitals from gray states had no available price transparency data for this telemedicine code. 
Acknowledgements: Data access and responsibility: Ms. Wu and Dr. Xiao had full access to all the data in the study and takes responsibility for the integrity of the data and the accuracy of the data analysis.

Shannon S. Wu, BA ${ }^{1}$

Vinay K. Rathi, $M D, M B A^{2}$

Joseph S. Ross, MD, MHS ${ }^{3}$

Rosh K.V. Sethi, MD, MPH

Roy Xiao, $M D, M S^{2}$

${ }^{1}$ Cleveland Clinic Lerner College of Medicine,

Cleveland, $\mathrm{OH}$, USA

${ }^{2}$ Department of Otolaryngology - Head and Neck Surgery, Massachusetts Eye and Ear,

243 Charles Street, Boston, MA 02114, USA

${ }^{3}$ Section of General Medicine, Department of Internal Medicine, Yale University School of Medicine,

New Haven, CT, USA

${ }^{4}$ Division of Otolaryngology - Head and Neck Surgery, Department of Surgery, Brigham and Women's Hospital,

Boston, MA, USA

Corresponding Author: Roy Xiao, MD, MS; Department of Otolaryngology - Head and Neck Surgery, Massachusetts Eye and Ear, 243 Charles Street, Boston, MA 02114, USA (e-mail: roy_xiao@meei.harvard.edu).

Author Contribution Ms. Wu and Dr. Xiao were responsible for the conception and design of this work, primary drafting of the manuscript, and acquisition of data and statistical analysis. All authors participated in the analysis and interpretation of the data and critically revised the manuscript for important intellectual content.

\section{Declarations:}

The authors assume full responsibility for the accuracy and completeness of the ideas presented.

Conflict of Interest: Ms. Wu, Dr. Rathi, Dr. Sethi, and Dr. Xiao have no conflicts of interest to report. Dr. Ross has received research support through Yale University from Johnson \& Johnson to develop methods of clinical trial data sharing, from the Food and Drug Administration to establish the Yale-Mayo Clinic Center for Excellence in Regulatory Science and Innovation (CERSI) program (U01FD005938), from the Medical Device Innovation Consortium as part of the National Evaluation System for Health Technology Coordinating Center (NESTcc), from the Agency for Healthcare Research and Quality (R01HSO22882), from the National Heart, Lung and Blood Institute of the National Institutes of Health (NIH) (RO1HSO25164, RO1HL144644), and from the Laura and John Arnold Foundation to establish the Good Pharma Scorecard at Bioethics International and to establish the Collaboration for Research Integrity and Transparency (CRIT) at Yale University.

\section{REFERENCES}

1. Volk J, Palanker D, O’Brien M, Goe C. States' Actions to Expand Telemedicine Access During COVID-19 and Future Policy Considerations. The Commonwealth Fund. Published 2021. . https://www. commonwealthfund.org/publications/issue-briefs/2021/jun/states-actions-expand-telemedicine-access-covid-19

2. Bestsennyy O, Gilberrt G, Harris A, Rost J. Telehealth: A quarter-trilliondollar post-COVID-19 reality? McKinsey \& Company. Published 2021 . . https://www.mckinsey.com/industries/healthcare-systems-and-services/our-insights/telehealth-a-quarter-trillion-dollar-post-covid-19reality

3. Xiao R, Rathi VK, Gross CP, Ross JS, Sethi RK V. Payer-Negotiated Prices in the Diagnosis and Management of Thyroid Cancer in 2021. JAMA. 2021;326(2):184-185. doi:https://doi.org/10.1001/jama.2021.8535

4. H.R.3684 - Infrastructure Investment and Jobs Act. Published 2021. Accessed September 20, 2021. https://www.congress.gov/bill/117th-congress/house-bill/3684

5. U.S. Department of Health \& Human Services. Biden-Harris Administration Invests over \$19 Million to Expand Telehealth Nationwide and Improve Health in Rural, Other Underserved Communities. Published 2021. Accessed October 12, 2021. https://www.hhs.gov/about/news/2021/ 08/18/biden-harris-administration-invests-over-19-million-expand-telehealth-nationwide-improve-health-rural.html

6. Uscher-Pines L, Sousa J, Jones M, et al. Telehealth Use Among Safety-Net Organizations in California During the COVID-19 Pandemic. JAMA. 2021;325(11):1106-1107. doi:https://doi.org/10.1001/jama.2021.0282

Publisher's Note: Springer Nature remains neutral with regard to jurisdictional claims in published maps and institutional affiliations. 